\title{
Relé de salto de vetor no SIMULINK: implementação da técnica de anti-ilhamento em sistemas de geração distribuída
}

\section{Vector jump relay on SIMULINK: implementation of anti-islanding techniques in distributed generation systems}

\author{
Alcedir Luis Finkler ${ }^{\varpi}, 1$, Luana Obregon ${ }^{\varpi, 1}$, Maurício de Campos ${ }^{\varpi}, 1$, João Manoel \\ Lenz $^{\varpi, 1}$, Paulo Sérgio Sausen ${ }^{\varpi, 1}$, Airam T. Z. Romcy Sausen ${ }^{\varpi, 1}$ \\ ${ }^{1}$ Doutorando em Modelagem Matemática, Universidade Regional do Noroeste do Estado do Rio Grande do Sul-UNIJUI, \\ ${ }^{2}$ Mestranda em Modelagem Matemática, Universidade Regional do Noroeste do Estado do Rio Grande do Sul-UNIJUI, \\ ${ }^{3}$ Departamento de Ciências Exatas e Engenharias, Universidade Regional do Noroeste do Estado do Rio Grande do \\ Sul-UNIJUI \\ *alcedir.finkler@iffarroupilha.edu.br; luana.obregon@sou.unijui.edu.br; campos@unijui.edu.br; joao.dasilva@unijui.edu.br; \\ sausen@unijui.edu.br; airam@unijui.edu.br...
}

Recebido: 09/09/2020. Revisado: 26/06/2021. Aceito: 08/09/2021.

\section{Resumo}

A crescente demanda por energia elétrica tem incentivado consumidores a investirem em geradores conectados junto às instalações consumidoras. Esse tipo de geração é conhecido como geração distribuída (GD). A GD apresenta vantagens, porém pode trazer alguns inconvenientes como o risco de alimentar um trecho de rede durante uma manutenção (operação ilhada). Para impedir que isso ocorra, as GDs devem possuir proteções que permitam identificar a perda de conexão com a rede. Entre os principais métodos de identificação de ilhamento estão os relés de salto de vetor. Estudos direcionados a detecção de ilhamento podem ser realizados utilizando diversos softwares, entre eles, pode-se citar, o MATLAB/SIMULINK. Porém, não há na literatura um documento que descreva com clareza como implementar uma simulação de deteç̧ão de ilhamento pelo método de salto de vetor utilizando MATLAB/SIMULINK. Esse trabalho tem como contribuição fornecer uma orientação detalhada dessa implementação utilizando máquina síncrona servindo como guia para alunos que desejam iniciar estudos em geração distribuída.

Palavras-Chave: Geração distribuída; Gerador Síncrono; Ilhamento; Perda de rede; Salto de Vetor.

\begin{abstract}
The growing demand for electricity has encouraged consumers to invest in generators connected to consumer facilities. This type of generation is known as distributed generation (DG). DG has advantages, but it can bring some inconveniences, such as the risk of feeding a section of the network during maintenance (islanded operation). To prevent this from happening, as the DGs must have protections that must identify the loss of connection to the network. Among the main methods of island detection are vector jump relays. Studies aimed at islanding detection can be performed using different softwares, among them, one can mention, MATLAB/SIMULINK. However, there is no document in the literature that clearly describes how to implement a simulation of island detection using the vector jump method on MATLAB/SIMULINK. This work contributes to provide a detailed orientation of this implementation using a synchronous machine serving as a guide for students who start studies in distributed generation.
\end{abstract}

Keywords: Distributed Generation; Islanding; Loss of mains; Synchronous generator; Vector Shift Relay. 


\section{Introdução}

A expansão industrial, o crescimento populacional e o desenvolvimento tecnológico, apresentam, como contrapartida, um aumento na demanda por energia elétrica. A matriz elétrica brasileira, que, conforme EPE (2019), dispõe de $66,6 \%$ da oferta interna proveniente de fontes hídricas, normalmente necessitando de grandes represas para armazenamento de água, longas linhas de transmissão para que a energia alcance aos centros consumidores, nos últimos anos tem sido suplementada por sistemas de geração de pequeno porte, instaladas próximas às unidades de consumo. Esse tipo de geração é chamado de geração distribuída (GD).

No Brasil, a resolução 482 da ANEEL (2012) que definiu normas claras sobre a GD permitiu a expansão desses sistemas. Além disso, para garantir a segurança dos trabalhadores que operam as redes de distribuição de energia, a norma IEEE 1547 define que, tão logo ocorra uma perda de conexão da GD com o sistema interligado de potência, a geração deverá ser capaz de detectar essa perda e automaticamente desconectar o gerador do sistema (IEEE, 2005). Essa proteção é conhecida como anti-ilhamento.

A norma IEEE 1547 ainda classifica as unidades geradoras em três tipos sendo: geradores de indução, geradores síncronos e inversores. Dentre esses, a maior preocupação são os geradores síncronos, uma vez que, esses possuem controladores de frequência e tensão que permitem que esses geradores continuem operando com os valores de tensão e frequência nominais da rede local mesmo com a perda de conexão com o sistema de potência (IEEE, 2005). No Brasil, a Agência Nacional de Energia Elétrica (ANEEL), por meio da lei $n^{\circ} 9.648 / 1998$ estabelece as condições de acesso aos sistemas de distribuição de energia elétrica a partir de concessionárias, permissionário ou autorizado. As principais normas e procedimentos relacionados são, o Módulo 3 dos procedimentos de distribuição, PRODIST, publicado pela ANEEL (2017), e a resolução normativa 482 da ANEEL (2012).

A resolução 482, além de definir o modelo em que será realizada a compensação da energia produzida, define as condições para que os produtores possam se conectar ao sistema de distribuição de energia. Em seu artigo terceiro, esta resolução, define que é responsabilidade das distribuidoras elaborar as normas técnicas que serão utilizadas para tratar do acesso a microgeração distribuída (até $75 \mathrm{~kW}$ ). Nesse sentido, como exemplo, pode ser citada a Companhia Paranaense de Energia, COPEL, que, em atendimento ao artigo terceiro da resolução 482 de 2012 da ANEEL, disponibiliza a normativa NTC 905200 . O documento, em seu item 5.2.1d justifica a importância dos relés para detecção de ilhamento e define a necessidade de implementação dessa função por métodos conhecidos como: taxa de variação da frequência (ANSI 81df/dt), e relé de salto de vetor (ANSI 78) (COPEL, 2018).

Laaksonen et al. (2018) descreve que a detecção de ilhamento pode ser realizada de maneira rápida utilizando relés de salto de vetor. Em seu trabalho cita o ajuste do ângulo de atuação do relé de salto de vetor utilizado nas simulações com valores de $+1^{\circ} \mathrm{e}-2^{\circ}$. As simulações são realizadas utilizando software PSCAD. Não é apresentada a forma de implementação da função de salto de vetor.
Freitas and Xu (2004) realiza uma investigação sobre a falsa operação de relés de salto de vetor. Descreve que em sua simulação foram utilizados ajustes no relé de $5^{\circ}, 10^{\circ}$ e $15^{\circ}$ considerando serem esses ajustes típicos de relés de salto de vetor. No documento é descrito o procedimento para obter o tempo de operação de relés de salto de vetor, porém não é mostrado de forma clara como implementar essa simulação.

Freitas et al. (2004) descreve que os relés de salto de vetor disponíveis no mercado realizam a medição do tempo de duração de cada semiciclo da rede, e comparam esse tempo de duração ao período do semiciclo anterior. Um aumento ou redução na duração do período será identificado como um salto de vetor provocando a atuação do relé. Descreve que os ajustes típicos para essa proteção são de $2^{\circ}$ a $20^{\circ}$. No artigo não é ilustrada de forma clara como implementar a simulação.

Kandakatla et al. (2015), descreve que relés de salto de vetor usualmente medem a duração de cada semiciclo da tensão da rede e comparam com a duração do semiciclo anterior. Em situação de ilhamento o tempo de cada semiciclo será alterado para mais ou para menos dependendo da parcela de carga ilhada ser superior ou inferior ao valor da potência gerada pela GD. Em seu trabalho descreve a implementação de um algorítimo realizando a função de um relé de salto de vetor. $\mathrm{O}$ trabalho é desenvolvido com o software PSCAD.

Para o desenvolvimento de estudos relacionados a detecção de ilhamento diferentes softwares podem ser utilizados. Rossiter (2010) realiza uma análise do uso do software MATLAB/SIMULINK afirmando que esta ferramenta é amplamente utilizada tanto na indústria como no ambiente acadêmico. Esse, apresenta opiniões de alunos quanto ao uso justificando a sua utilização voltada à pesquisa e educação. A validação do método proposto foi realizada utilizandose o programa MATLAB/SIMULINK. Esse programa tem sido amplamente utilizado em pesquisas voltadas à proteção no SEP como em Rostami et al. (2017), Admasie et al. (2019), Rostami, Olamaei and Abdi (2019), Rostami, Jalilian, Hagh, Muttaqi and Olamaei (2019), Taheri Kolli and Ghaffarzadeh (2020), Alam and Saha (2020), Abdi et al. (2020), Markovic et al. (2021), Protection strategy for fault detection in inverter-dominated low voltage AC microgrid (2021), Khalid and Shobole (2021).

Em um levantamento bibliográfico realizado a partir da ferramenta de busca IEEE Xplore utilizando os termos mais frequentes em artigos relacionados a proteção antiilhamento e detecção por salto de vetor ("islanding" e "vector shift", "islanding" e "vector surge", "loss of mains" e "vector shift", "loss of mains" e "vector surge") foram encontrados as referências listadas na Tabela 1.

Analisando o conteúdo da Tabela 1 é possível observar que há apenas um trabalho desenvolvido em MATLAB/SIMULINK, relacionado a ensaios de ilhamento, utilizando o método de salto de vetor, que pode ser utilizado como referência para introdução a este assunto a nível acadêmico.

Banu et al. (2014) apresenta uma proposta de implementação de um relé de salto de vetor conforme ilustrado na Fig. 1. Descreve que o circuito calcula a duração de cada período realizando a divisão do tempo de cada ciclo pelo número de ciclos. Esse valor é comparado a um valor pré definido. Caso esse tempo ultrapasse os valores ajusta- 
Tabela 1: Publicações disponíveis na IEEE Xplore

\begin{tabular}{|c|c|c|}
\hline Título & Autor & Software* \\
\hline $\begin{array}{c}\text { Protection control strategy for effectively interconnecting and islanding distributed } \\
\text { energy resources during grid disturbances }\end{array}$ & Xavier(2019) & ** \\
\hline Implications for the Rate of Change of Frequency on an Isolated Power System & O'Donovan(2019) & ** \\
\hline Islanding Detection During Intended Island Operation of Nested Microgrid & Laaksonen(2018) & PSCAD \\
\hline $\begin{array}{l}\text { Wide Area Phase Angle Measurements for Islanding Detection - An Adaptive Nonlinear } \\
\text { Approach }\end{array}$ & $\operatorname{Liu}(2016)$ & DigSilent \\
\hline $\begin{array}{l}\text { Grid code compatible islanding detection schemes using traditional passive methods } \\
\text { Synchrophasor-Based Islandina Detection for Distributed Generation Systems Using }\end{array}$ & Laaksonen(2016) & PSCAD \\
\hline Systematic Principal Component Analysis Approaches & Guo(2015) & ** \\
\hline Islanding Detection Based on Probabilistic PCA with Missing Values in PMU Data & $\operatorname{Liu}(2014)$ & ** \\
\hline $\begin{array}{l}\text { A Study on Anti-Islanding Detection Algorithms for Grid-Tied Photovoltaic Systems } \\
\text { A Composite Method for Islanding Detection Based on Vector Shift and Frequency }\end{array}$ & Banu(2014) & SIMULINK \\
\hline Variation & $\mathrm{Hou}(2010)$ & PSCAD \\
\hline Dispersed generation in MV networks: performance of anti-islanding protections & Delfanti(2010) & DigSilent \\
\hline $\begin{array}{l}\text { Design and Implementation of an Anti-Islanding Protection Strategy for Distributed } \\
\text { Generation involving Multiple Passive Protection }\end{array}$ & Foss (2009) & ** \\
\hline $\begin{array}{c}\text { A Practical Method for Assessing the Effectiveness of Vector Surge Relays for } \\
\text { Distributed Generation Applications }\end{array}$ & Freita & $* *$ \\
\hline False Operation of Vector Surge Relays & Freitas(2004) & ** \\
\hline
\end{tabular}

\footnotetext{
* Software utilizado nas simulações;
}

** Não informado.

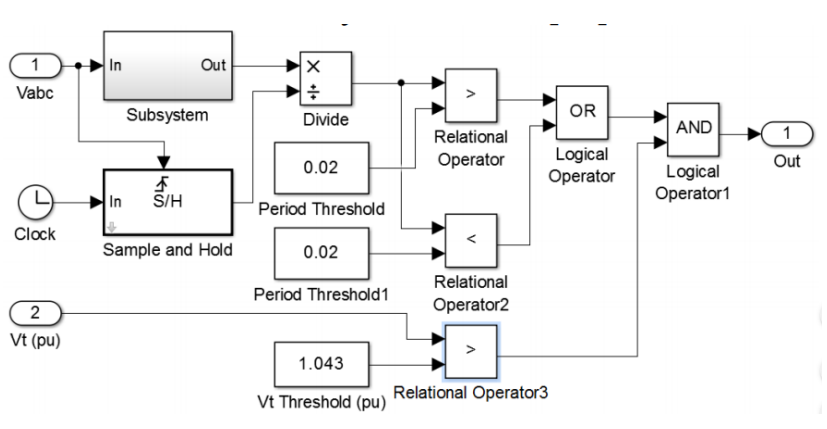

Figura 1: Relé de salto de vetor

dos é considerada uma condição de ilhamento. O modelo apresentado por Banu et al. (2014), é a única proposta de diagrama para ser implementado no SIMULINK encontrado com os métodos apresentados, porém o modelo aqui proposto está mais adequado ao princípio de funcionamento descrito por Kandakatla et al. (2015), Freitas et al. (2004), Freitas and $\mathrm{Xu}$ (2004) e Laaksonen et al. (2018), uma vez que, no modelo proposto nesse trabalho, o deslocamento é calculado comparando o período de cada ciclo ao período imediatamente anterior. Também no modelo aqui proposto é considerada a desabilitação do relé em caso de um afundamento de tensão.

Neste contexto, o presente trabalho tem como objetivo principal disponibilizar uma fonte de orientação detalhada a estudantes quanto a forma de implementar o sistema de controle de uma máquina síncrona e como implementar um circuito para detecção de salto de vetor utilizando o software MATLAB/SIMULINK.

Foram levadas algumas considerações para o desenvolvimento deste trabalho, dentre elas se destacam: as particularidades descritas com relação à máquinas síncronas; a importância dos métodos de detecção de ilhamento; o fato de companhias de distribuição, a exemplo da COPEL, exigirem a implementação pelo método de salto de vetor; a popularidade do uso do software MATLAB/SIMULINK no ambiente acadêmico, associada a falta de uma documentação clara que possa servir como guia a alunos que desejam iniciar estudos direcionados a GD.

O presente artigo disponibiliza uma orientação clara quanto ao método de simulação e orientações quanto aos procedimentos que devem ser avaliados na identificação do ilhamento. Esse artigo não foi desenvolvido com foco à uma contribuição para indústria, mas sim, para fornecer um suporte introdutório aos universitários que iniciam seus estudos no contexto da geração distribuída.

$O$ restante deste artigo está organizado da seguinte forma: na Seção 2 é descrito o princípio básico de funcionamento dos relés de salto de vetor. Na Seção 3 é apresentada a implementação do relé de salto de vetor a partir do MATLAB/SIMULINK. Já na Seção 4 é descrita a forma de implementação de uma máquina síncrona, o controle de excitação é apresentado na Seção 5 . Os resultados das simulações realizadas são apresentados na Seção 6. Na Seção 7 são apresentadas as conclusões deste trabalho.

\section{Princípio básico do relé de salto de vetor}

Kandakatla et al. (2015) descreve o princípio básico de funcionamento de um relé de salto de vetor, utilizando como referência a Fig. 2 . Nesse diagrama, $\bar{E}_{I}$ representa a tensão interna da máquina síncrona, $\Delta \bar{V}$ representa a queda de tensão na reatância da máquina síncrona $X_{d}$ em função da corrente $\bar{I}_{S}, \bar{V}_{T}$ representa a tensão no ponto de acoplamento entre o gerador e a rede, $\bar{I}_{G r}$ representa a corrente proveniente da rede. $C B$ é o interruptor que será utilizado para seccionamento da rede deixando o gerador alimentando uma parcela da carga ilhada. VS representa o interruptor que deverá ser conectado à saída do gerador para desconectá-lo no momento em que o circuito antiilhamento detecte a perda de conexão com a rede. 


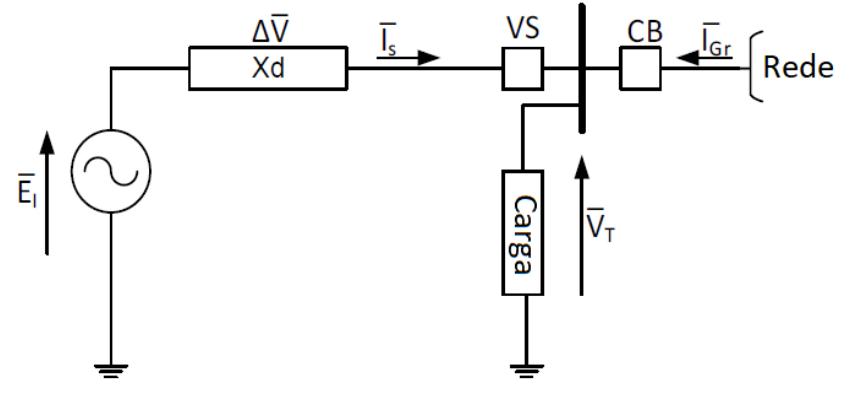

Figura 2: Gerador síncrono conectado paralelo a rede Adaptado de Kandakatla et al. (2015)

Kandakatla et al. (2015) descreve que, durante o período em que o gerador opera em paralelo a rede, a carga conectada ao ponto de conexão comum será alimentada por $\bar{I}_{S}+\bar{I}_{G r}$. A tensão interna da máquina $\bar{E}_{I}$ estará defasada da tensão no ponto de conexão $\bar{V}_{T}$ em um ângulo $\theta$. No momento em que houver uma perda de conexão com a rede, simulado pela abertura do interruptor $C B$, a carga passa a ser alimentada exclusivamente por $\bar{I}_{S}$, a tensão no ponto de conexão passa a ser representada por $\bar{V}_{T}^{\prime}$, logo, haverá uma alteração no valor do ângulo de defasagem formado entre a tensão interna da máquina e a tensão nos terminais. Essa alteração será representada por $\Delta \theta$. A Fig. 3a representa os vetores das tensões no momento anterior a abertura do interruptor $\mathrm{CB}$, enquanto a Fig. 3 b representa os vetores das tensões no momento posterior a abertura.

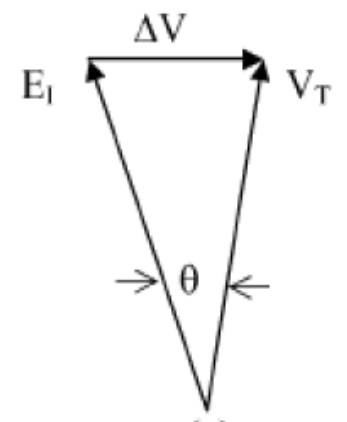

(a)

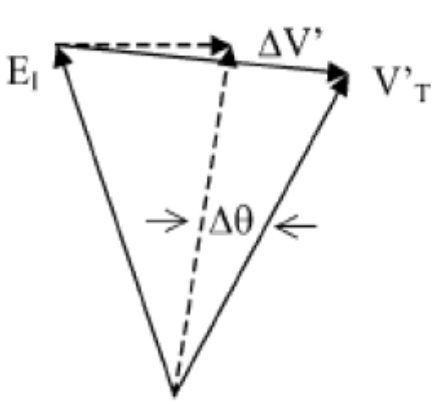

(b)
Figura 3: Diagrama fasorial Adaptado de Kandakatla et al. (2015)

Hou et al. (2008) descreve que, no momento da perda de conexão com a rede, haverá uma variação no ângulo formado entre a tensão interna e a tensão nos terminais da máquina anteriormente descrita, provocando um deslocamento no perfil da tensão no ponto de conexão comum conforme ilustrado na Fig. 4. Esse deslocamento é chamado de salto de vetor.

Freitas et al. (2005) descreve um procedimento de implementação para o relé de salto de vetor no qual, a cada um terço de ciclo é calculado o desvio entre o ângulo atual e ângulo do ciclo anterior. Na Fig. 5 é possível observar que no início do primeiro ciclo de rede é considerado o pri-

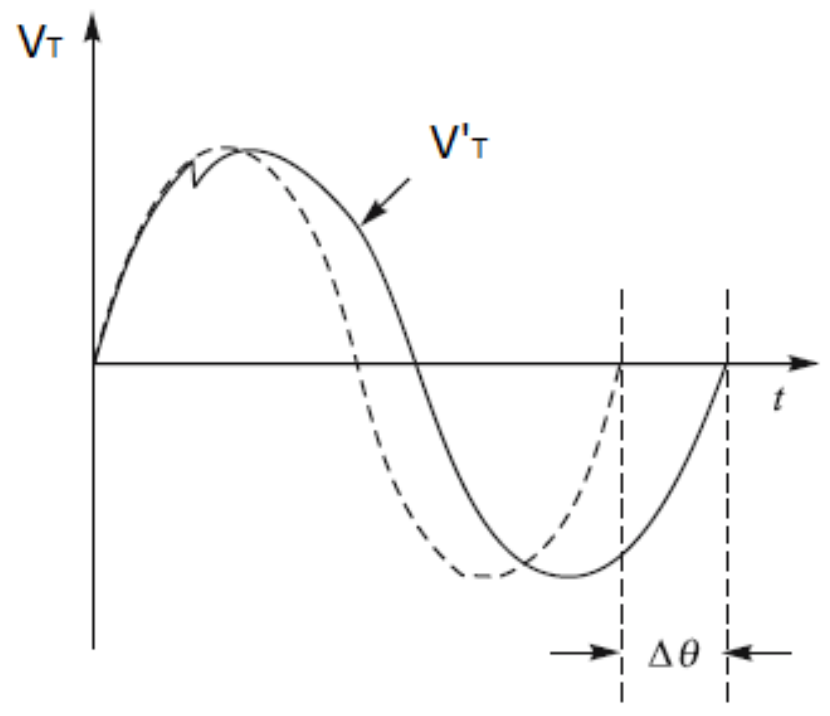

Figura 4: Tensão no ponto de acoplamento Adaptado de Hou et al. (2008)

meiro ciclo de integração. Até o quarto ciclo de integração foram realizadas três verificações do desvio angular. Ao iniciar o segundo ciclo de rede, terá inicio o quarto ciclo de integração no qual o calculo do desvio angular é reiniciado. Freitas et al. (2005) apresenta a necessidade de implementar uma função que desabilite o relé de salto de vetor quando a tensão da rede estiver abaixo de um determinado valor de referência, impedindo que o relé de salto de vetor atue indevidamente durante, por exemplo, o momento de partida dos geradores ou em casos de curtocircuito, causando instabilidade ao sistema. Para isso, é adicionado ao código de programação disponível no anexo desse documento, uma função que desabilita o relé quando a tensão da rede estiver com valor inferior a $90 \%$.

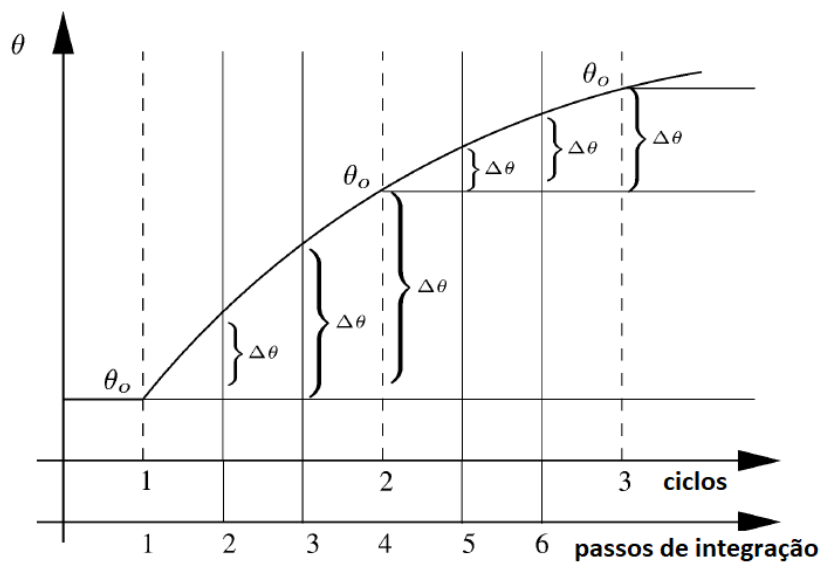

Figura 5: Curva de integração relé Adaptado de Freitas et al. (2005) 


\section{Implementação do relé de salto de vetor no Simulink}

A implementação do relé de salto de vetor descrita nesse artigo foi realizada utilizando um bloco "S-Funtion Level 2 " do SIMULINK conforme ilustrado na Fig. 6. Esse bloco executa um programa desenvolvido no MATLAB. O programa utilizado está disponível no Anexo A. Esse bloco foi construído com fins didáticos de facilitar a compreensão da implementação do relé de salto de vetor utilizando MATLAB/SIMULINK.

Para simulação considerou-se um sistema balanceado, com o seccionamento da rede sendo realizado nas três fases simultaneamente. O relé de salto de vetor foi implementado apenas na fase A. Para calcular o período de duração de cada semiciclo de rede é tomado como referência o momento em que a tensão de cada uma das fases passa por zero. Logo, o primeiro bloco corresponde a um detector de passagem por zero. Seu terminal de saída passa a nível lógico alto sempre que a tensão da fase $A$ atinge um valor de zero volts. Um bloco de atraso na desenergização é implementado para solucionar problemas com passo de calculo. A segunda entrada do bloco "S-Funtion Level 2 "é conectada ao relógio para obter o tempo da simulação e determinar o momento em que ocorre cada passagem por zero. A terceira entrada do bloco "S-Funtion Level 2"é utilizada para definir o ângulo de deslocamento que deverá provocar a atuação do dispositivo. A quarta entrada do bloco é conectada a medição da tensão de linha.

O funcionamento do bloco "S-Funtion Level 2 "será definido por meio da programação realizada em código $M$, apresentada no anexo A descrito a seguir. Para determinar o período de cada semiciclo são utilizadas as variáveis "tempo1"e "tempo2". A cada passagem por zero, ou seja, no momento em que a entrada 1 receber um sinal do detector de passagem por zero, o programa irá mover o valor previamente salvo na variável "tempo1"para a variável "tempo2", em seguida será atualizado o valor da variável "tempo1"com o tempo em que ocorreu a passagem por zero. Logo, a variável "tempo1"possui a informação do instante de tempo em que ocorreu a última passagem por zero e, a variável "tempo2", possui a informação do tempo em que ocorreu a passagem por zero anterior. Um vez conhecido o momento das duas últimas passagens por zero, é possível calcular o período do último semiciclo, subtraindo o valor do tempo registrado na variável "tempo2"do valor de tempo registrado na variável "tempo1". Esse período será considerado como período atual. A cada nova passagem por zero, o valor do período atual é salvo na variável "periodo_anterior", e o valor do período de duração do último semiciclo da tensão da rede será atualizado na variável "periodo_atual". Uma vez conhecido o valor do período do último semiciclo da rede e o período anterior, esses poderão ser comparados. Próximo passo é verificar se existe variação de tempo entre esses períodos. Isso é realizado subtraindo o valor da variável "periodo_atual"da variavel "periodo_anterior". O resultado da subtração é registrado na variável "variacao_periodo". Essa variável armazena o valor em segundos da variação de tempo ocorrida entre o último semiciclo da rede e o semiciclo anterior. Dividindo esse valor de variação em segundo pelo período do último semiciclo em segundo, é possível obter o valor da

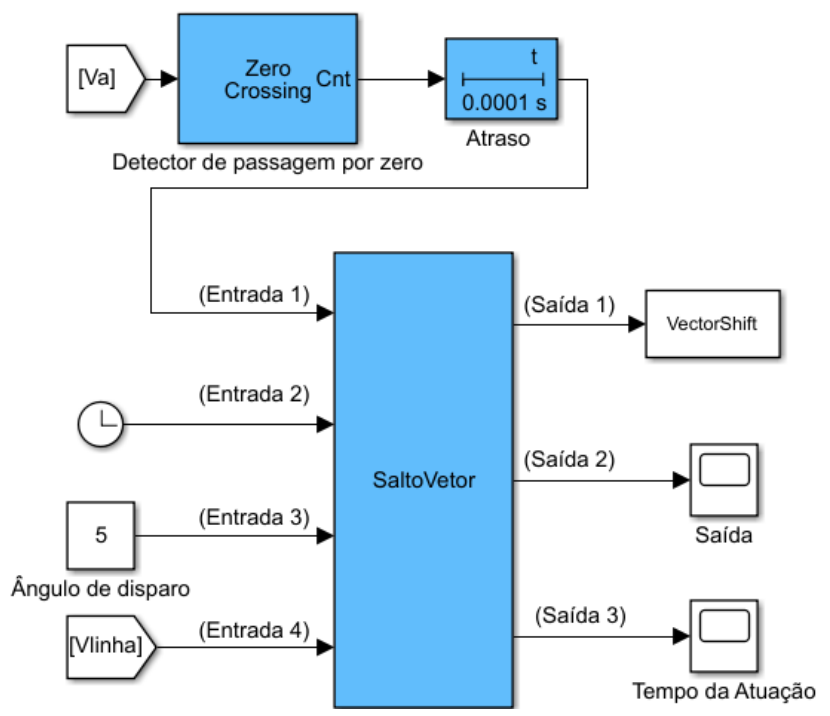

Figura 6: Diagrama do relé de salto de vetor no Simulink

variação em percentual. Sendo que, cada semiciclo da rede correspondente a $180^{\circ}$ elétricos, multiplicando a variação do período em percentual por 180 é obtida a variação em graus elétricos $(\Delta \theta)$. Essa informação será disponibilizada na saída 1 do bloco "S-Funtion Level 2".

O valor da variação de ângulo obtido a cada interação é comparado ao valor do ângulo de disparo definido por meio da entrada 3 do bloco "S-Funtion Level 2". Quando for detectada uma variação angular superior ao valor ajustado como referência na entrada 3, haverá o acionamento da saída 2. Essa saída poderá ser utilizada para realizar a desconexão do gerador. A saída 3 fornece o tempo em que houve o disparo do relé de salto de vetor.

\section{Configuração do Gerador}

A norma IEEE Std 1547.1-2005 descreve os diversos procedimentos a serem ensaiados com GDs como, estabilidade de temperatura, resposta a condições anormais de tensão, resposta a condições anormais de frequência, ensaios de sincronismo entre outros. A secção 5.7 dessa norma é destinada a ensaios para ilhamento não intencional. No capítulo 5.7.2.2 é definido o procedimento para ensaio. Um dos testes a ser realizado consiste em configurar a máquina para máxima potência ativa e ajustar a carga de forma a obter uma corrente no interruptor "CB"equivalente a $2 \%$ da corrente do gerador. Esses ensaios tem como objetivo verificar a capacidade da proteção anti-ilhamento atuar diante à dinâmica do circuito responsável pelo controle da corrente de campo. O teste é considerado satisfatório se a conexão for cessada dentro do limite de tempo de 2 segundos após a abertura do interruptor "CB"(IEEE, 2005).

Em seguida o teste deve ser repetido com máxima potência ativa e fator de potência atrasado; máxima potência ativa e fator de potência adiantado (IEEE, 2005). Para essa simulação, a potência mecânica é considerada como contante igual a 1 p.u. O diagrama utilizado para as simulações está ilustrado na Fig. 7.

Após a atuação do interruptor CB para ensaio do ilha- 


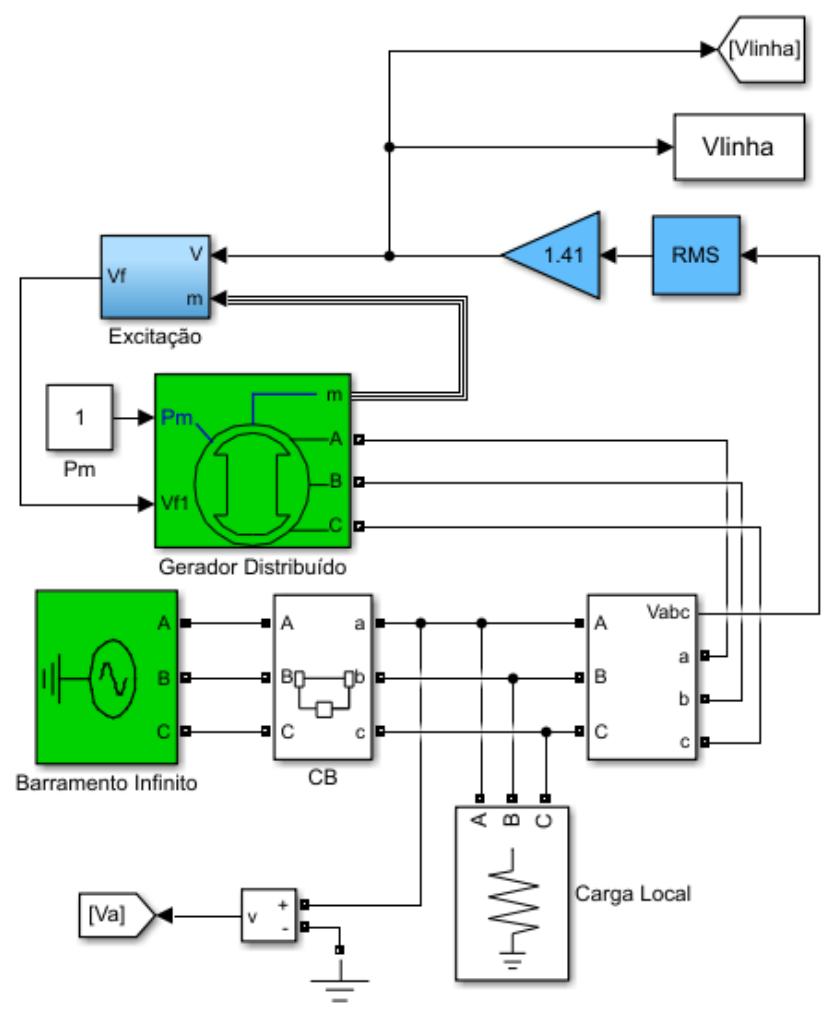

Figura 7: Diagrama do gerador conectado ao barramento infinito

mento, a rede é desconectada do circuito, permanecendo o gerador ilhado energizando a carga local. Logo, para análise do comportamento do ramal ilhado, as informações referentes a rede, a exemplo do nível de curto-circuito, podem ser desprezadas. Para realização das simulações o interruptor CB é configurado para abertura após decorridos 3 segundos do início da simulação. A carga local é configurada como potência constante. Para realização das três simulações apresentadas nesse trabalho, foram atribuídas à carga local potências de: $130 \%, 150 \%$ e $180 \%$ da potência gerada pelo gerador distribuído. O bloco de medição de tensão deve ser configurado para fornecer valores em p.u.. Para o gerador utiliza-se o bloco "Synchronous Machine p.u. Standard" definindo o modelo pré-configurado 15 disponível na biblioteca do SIMULINK $(6 \mathrm{oHz}, 460 \mathrm{~V}, 37.5 \mathrm{kVA}$, 1800RPM). Para realização das simulações é necessário acrescentar o bloco "powergui". Esse é configurado para o tipo de simulação em modo contínuo. Na guia de configurações é definido que a simulação será executada com passo variável. O método de solução, na guia de configurações, deve ser selecionado para opção "automática".

\section{Configuração da excitação}

Os geradores síncronos podem ser configurados no SIMULINK para operar em três diferentes formas, sendo como PQ (com valor de potência ativa e potência reativa definidos), PV (com valor de potência ativa e tensão nos terminais definidos), ou como "Swing" (gerador atua no

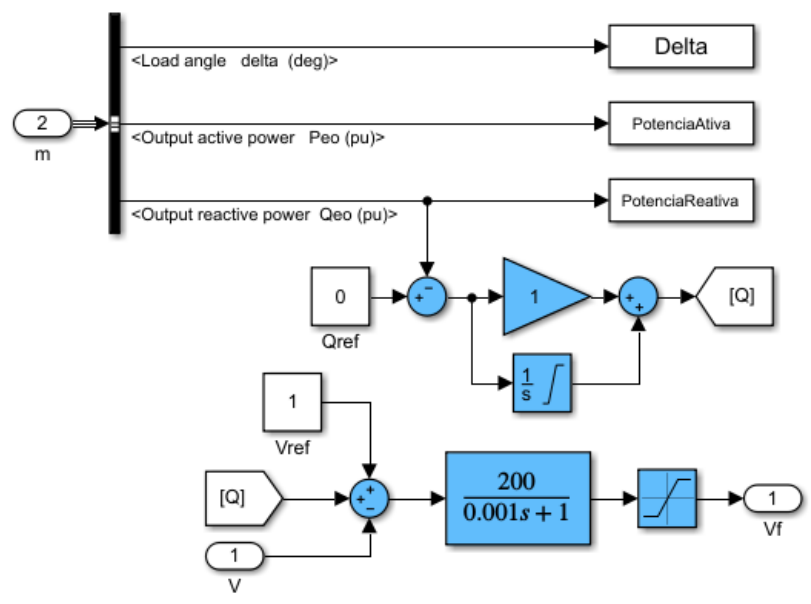

Figura 8: Diagrama da excitação do gerador

controle da frequência e tensão no ponto de conexão).

A norma IEEE Std 1547-2018, em seu capítulo 5.3.1, define que os geradores deverão ser configurados para operar com fator de potência constante e unitário, e, apenas poderão ser configurados para atuar no controle de tensão ou diferentes modos com autorização da distribuidora (IEEE, 2018). Logo, nas simulações os geradores deverão ser configurados como PQ especificando os valores de potência reativa como zero.

A norma IEEE Std 421.5-2016 fornece modelos matemáticos para simulações de sistemas de excitação de geradores síncronos trifásicos. Em seu capítulo 8.2 descreve o modelo ST1A para controle de excitação estática. No capítulo 13.7 descreve o controlador "VAR TYPE 2", utilizado para manter o fator de potência da geração constante (IEEE, 2016). O Anexo $\mathrm{H}$ dessa norma fornece os dados em p.u. para serem utilizados como referência nas simulações desses equipamentos. Na ?? é ilustrada a implementação do sistema de controle de excitação.

\section{Resultado das simulações}

O funcionamento do controle de excitação pode ser observado na Fig. 9. $O$ valor da potência reativa será mantido próximo a zero p.u..

Na Fig. 10 é apresentado o perfil da tensão no semiciclo em que ocorre o ilhamento. É possível observar que haverá um deslocamento no perfil da tensão de acordo com a potência ativa da carga que permanecerá ilhada.

Com a ocorrência do ilhamento aos 3 segundos, caso a carga ilhada possua potência ativa equivalente a potência gerada pela GD antes da ocorrência do ilhamento, o término desse semiciclo ocorrerá em 3,00833 segundos. Com potência ilhada $30 \%$ superior a potência do gerador antes do ilhamento, o término do semiciclo ocorrerá em 3,00846 segundos, 3,00858 segundos para $50 \%$ de acréscimo na carga e 3,00875 segundos para $80 \%$ de acréscimo na carga.

Para uma melhor visualização, na Fig. 11 é ilustrado o tempo em que ocorre a passagem por zero ao término do ciclo ilustrado na Fig. 10. 


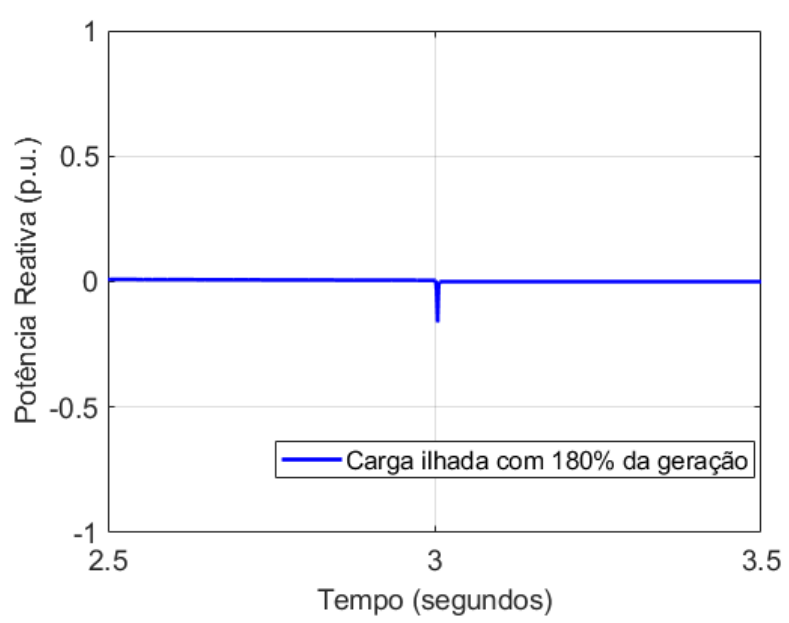

Figura 9: Potência reativa em simulação com carga ilhada equivalente a $180 \%$ da potência ativa gerada

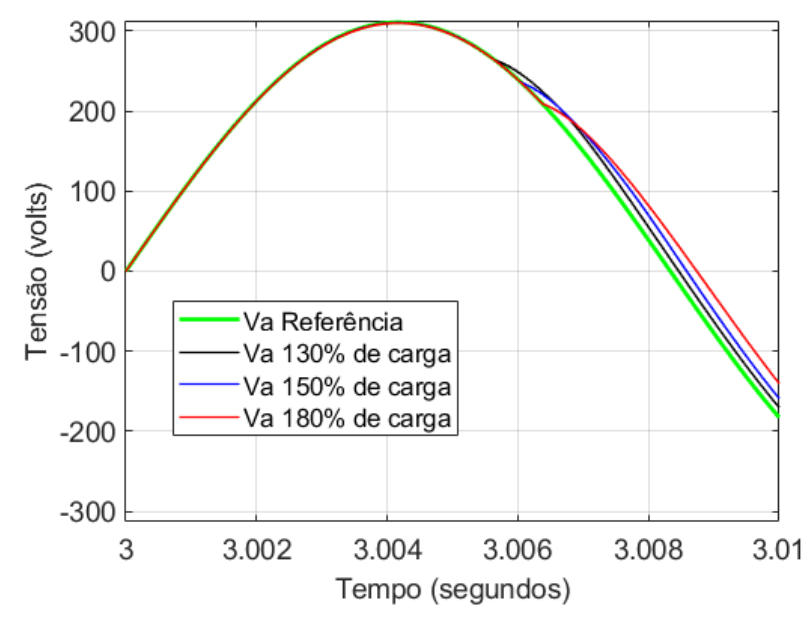

Figura 10: Perfil da tensão durante ilhamento

Logo, é possível obter o valor da variação de tempo no semiciclo em que ocorreu o ilhamento e ângulo de deslocamento. Esses valores estão ilustrados na Tabela 2.

Na Fig. 12 é apresentado o comportamento da saída 1 do bloco de detecção de ilhamento. Nessa saída é possível verificar o valor da variação de ângulo calculado pelo bloco "S-Funtion Level 2"para cada semiciclo da tensão da rede utilizada como referência.

\section{Conclusão}

Nesse artigo foi abordada importância dos métodos de detecção de ilhamento em sistemas com máquinas síncronas utilizando o método conhecido como salto de vetor. Foi descrito um procedimento para implementação do relé de salto de vetor utilizando o bloco do SIMULINK "S-Funtion Level 2". Esse trabalho serve como um suporte a estudantes e pesquisadores cuja área de interesse esteja voltada a

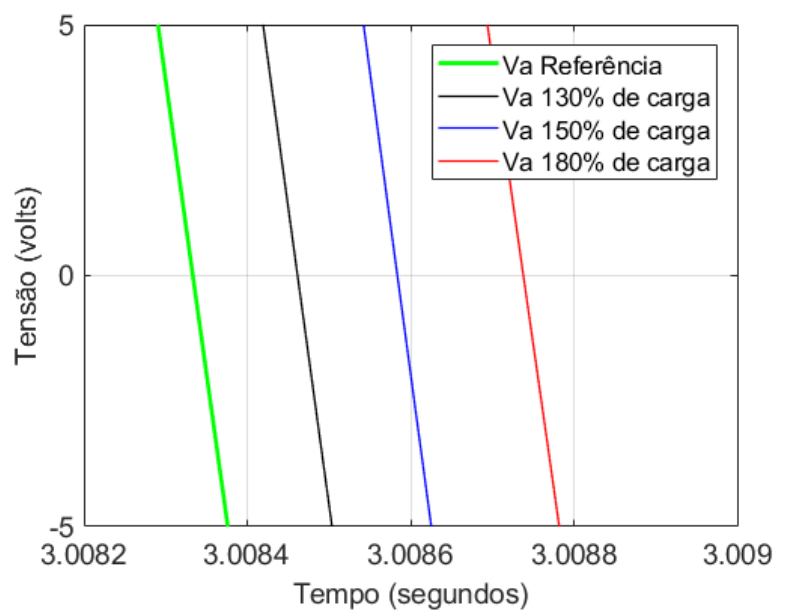

Figura 11: Passagem por zero após ilhamento

Tabela 2: Ângulo de deslocamento da tensão após ocorrência do ilhamento

\begin{tabular}{ccccc}
\hline$\Delta \mathrm{P}$ & tempo & $\Delta \mathrm{t}(\mathrm{ms})$ & $\Delta \mathrm{t}(\%)$ & $\Delta \theta$ \\
\hline $0 \%$ & $8,33 \mathrm{~ms}$ & $0 \mathrm{~ms}$ & $0 \%$ & $0^{\circ}$ \\
$30 \%$ & $8,46 \mathrm{~ms}$ & $0,13 \mathrm{~ms}$ & $0,0156 \%$ & $2,8^{\circ}$ \\
$50 \%$ & $8,58 \mathrm{~ms}$ & $0,25 \mathrm{~ms}$ & $0,03 \%$ & $5,4^{\circ}$ \\
$80 \%$ & $8,75 \mathrm{~ms}$ & $0,42 \mathrm{~ms}$ & $0,0504 \%$ & $9,07^{\circ}$ \\
\hline
\end{tabular}

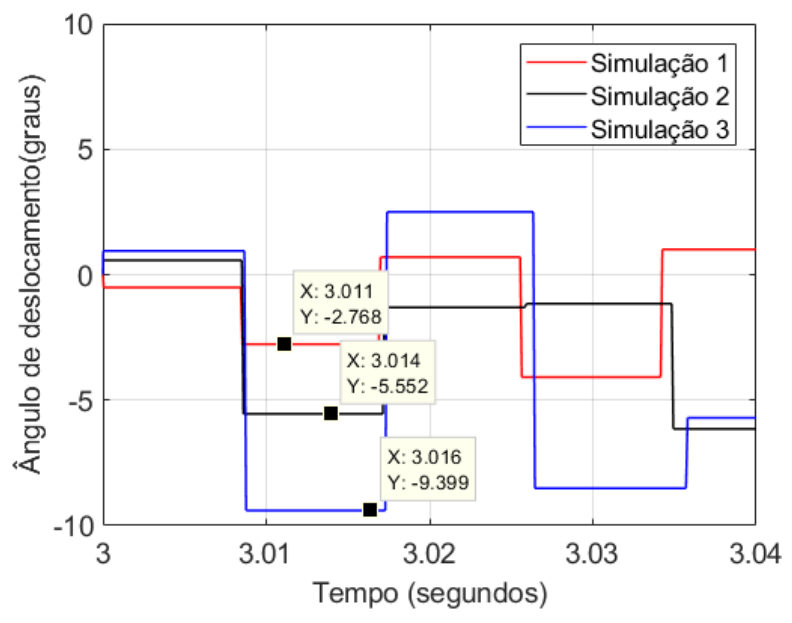

Figura 12: Deslocamento de vetor obtido com bloco de função

geração distribuída. Foi apresentado de forma detalhada todo o método de implementação de um gerador síncrono conectado ao barramento infinito, com controle de excitação e dispositivo para deteç̧ão de ilhamento utilizando relé de salto de vetor embasando as escolhas em normas técnicas e demais artigos relacionadas ao assunto. 


\section{Agradecimentos}

Ao Instituto Federal Farroupilha-Campus Santa Rosa pelo apoio e incentivo a qualificação profissional, e em especial ao Programa Institucional de Incentivo à Qualificação Profissional dos Servidores do IFFar (PIIQP).

\section{Referências}

Abdi, H., Rostami, A. and Rezaei, N. (2020). A novel passive islanding detection scheme for synchronoustype dg using load angle and mechanical power parameters, Electric Power Systems Research p. 106968. https: //doi.org/10.1016/j. epsr.2020.106968.

Admasie, S., Bukhari, S. B. A., Haider, R., Gush, T. and Kim, C.-H. (2019). A passive islanding detection scheme using variational mode decomposition-based mode singular entropy for integrated microgrids, Electric Power Systems Research 177: 105983. https://doi.org/10. 1016/j . epsr. 2019.105983.

Alam, M. R. and Saha, T. K. (2020). Detection of dgislanding employing decision boundary of polarization ellipse parameters, Electric Power Systems Research 188: 106536. https://doi.org/10.1016/j.epsr. 2020. 106536 .

ANEEL (2012). Resolução Normativa $n^{\circ}$ 482, de 17 de abril de 2012., ANEEL. Disponível em: http://www2 aneel gov . $\mathrm{br} /$ cedoc/ren2012482.pdf.

ANEEL (2017). Procedimentos de Distribuição de Energia Elétrica no Sistema Elétrico Nacional - PRODIST - Módulo 3., ANEEL. Disponível em: http://www2 . aneel.gov.br/ arquivos/PDF/Modulo3_Revisao_3_Retificacao_1.pdf.

Banu, I. V., Istrate, M., Machidon, D. and Pantelimon, R. (2014). A study on anti-islanding detection algorithms for grid-tied photovoltaic systems, 2014 International Conference on Optimization of Electrical and Electronic Equipment (OPTIM), pp. 655-660. https://doi.org/10. 1109/OPTIM. 2014.6850940.

COPEL (2018). Acesso de micro e minigeração distribuída ao sistema da Copel (com compensação de energia), COPEL. Disponível em: https://https://www.copel. com/hpcopel/root/sitearquivos2.nsf/verdocatual/ E59DF9E94B635F678325831D0047F719/〈protect \T1\ textdollarFILE/NTC905200_Rev04102018.pdf.

EPE (2019). Balanço Energético Nacional 2019: Ano base 2018, Empresa de Pesquisa Energética. Disponível em: http://www.epe.gov.br/ sites-pt/publicacoes-dados-abertos/publicacoes/ PublicacoesArquivos/publicacao-377/topico-494/ BEN\%202019\%20Completo\%20WEB. pdf.

Freitas, W., Huang, Z. and Xu, W. (2004). A practical method for assessing the effectiveness of vector surge relays for distributed generation applications, IEEE Power Engineering Society General Meeting, 2004, Vol. 1, p. 821. https://doi.org/10.1109/PES.2004.1372931.
Freitas, W. and Xu, W. (2004). False operation of vector surge relays, IEEE Transactions on Power Delivery 19(1): 436-438. https://doi.org/10.1109/TPWRD. 2003. 820412.

Freitas, W., Xu, W., Affonso, C. M. and Huang, Z. (2005). Comparative analysis between rocof and vector surge relays for distributed generation applications, IEEE Transactions on Power Delivery 20(2): 1315-1324. https : // doi.org/10.1109/TPWRD. 2004.834869.

Hou, M., Gao, H., Liu, B. and Zou, G. (2008). Vector shift method for islanding detection based on simulation test, Transactions of Tianjin University 14: 123-127. https : // doi.org/10.1007/s12209-008-0022-x.

IEEE (2005). IEEE standard conformance test procedures for equipment interconnecting distributed resources with electric power systems, IEEE Std 1547.1-2005 pp. 162. https ://doi .org/10.1109/IEEESTD . 2005. 96289.

IEEE (2016). IEEE recommended practice for excitation system models for power system stability studies, IEEE Std 421.5-2016 (Revision of IEEE Std 421.5-2005) pp. 1-207. https://doi .org/10.1109/IEEESTD. 2016.7553421.

IEEE (2018). IEEE standard for interconnection and interoperability of distributed energy resources with associated electric power systems interfaces, IEEE Std 15472018 (Revision of IEEE Std 1547-2003) pp. 1-138. https : //doi.org/10.1109/IEEESTD.2018.8332112.

Kandakatla, M., Laaksonen, H. and Bonela, S. (2015). Advanced vector shift algorithm for islanding detection. Disponível em http://cired.net/publications/ cired2015/papers/CIRED2015_0480_final.pdf.

Khalid, H. and Shobole, A. (2021). Existing developments in adaptive smart grid protection: A review, Electric Power Systems Research 191: 106901. https : //doi .org/ 10.1016/j. epsr.2020.106901.

Laaksonen, H., Hovila, P., Kauhaniemi, K. and Sirviö, K. (2018). Advanced islanding detection in grid interactive microgrids, Proceedings of the CIRED 2018 Ljubljana Workshop, AIM, Ljubljana, Slovenia. Disponível em https://www.cired-repository.org/ bitstream/handle/20.500.12455/1266/CIRED\%202018\% 20Ljubljana $\% 20 W S \% 20-\% 200056 \% 20-\% 2020734$. pdf? sequence $=1 \&$ is sllowed $=y$.

Markovic, U., Chrysostomou, D., Aristidou, P. and Hug, G. (2021). Impact of inverter-based generation on islanding detection schemes in distribution networks, Electric Power Systems Research 190: 106610. https: //doi.org/10.1016/j. epsr.2020.106610.

Protection strategy for fault detection in inverter-dominated low voltage AC microgrid (2021). Electric Power Systems Research 190: 106572. https: //doi .org/10.1016/j . epsr.2020.106572", author =.

Rossiter, J. A. (2010). Using matlab for teaching control design and analysis, UKACC International Conference on Control 2010, pp. 1-6. https://doi .org/10.1049/ic . 2010.0401. 
Rostami, A., Jalilian, A., Hagh, M. T., Muttaqi, K. and Olamaei, J. (2017). Islanding detection of distributed generation based on rate of change of exciter voltage and mechanical power with circuit breaker switching strategy, 2017 IEEE Industry Applications Society Annual Meeting pp. 1-8. https://doi.org/10.1109/IAS.2017.8101799.

Rostami, A., Jalilian, A., Hagh, M. T., Muttaqi, K. and Olamaei, J. (2019). Islanding detection of distributed generation based on rate of change of exciter voltage with circuit breaker switching strategy, IEEE Transactions on Industry Applications 55: 954-963. https ://doi .org/10. 1109/TIA. 2018. 2868547.

Rostami, A., Olamaei, J. and Abdi, H. (2019). Islanding detection of synchronous dg based on inherent feature extracted from mechanical power, Iranian Journal of Science and Technology, Transactions of Electrical Engineering 43: 919-928. https://doi.org/10.1007/ s40998-019-00193-8.

Taheri Kolli, A. and Ghaffarzadeh, N. (2020). A novel phaselet-based approach for islanding detection in inverter-based distributed generation systems, Electric Power Systems Research 182: 106226. https : //doi .org/ 10.1016/j. epsr.2020.106226.

\section{A Programa salto de vetor}

Para realizar a simulação no MATLAB/SIMULINK, com o bloco de função de segunda ordem, é necessário carregar o programa descrito na Fig. 13:

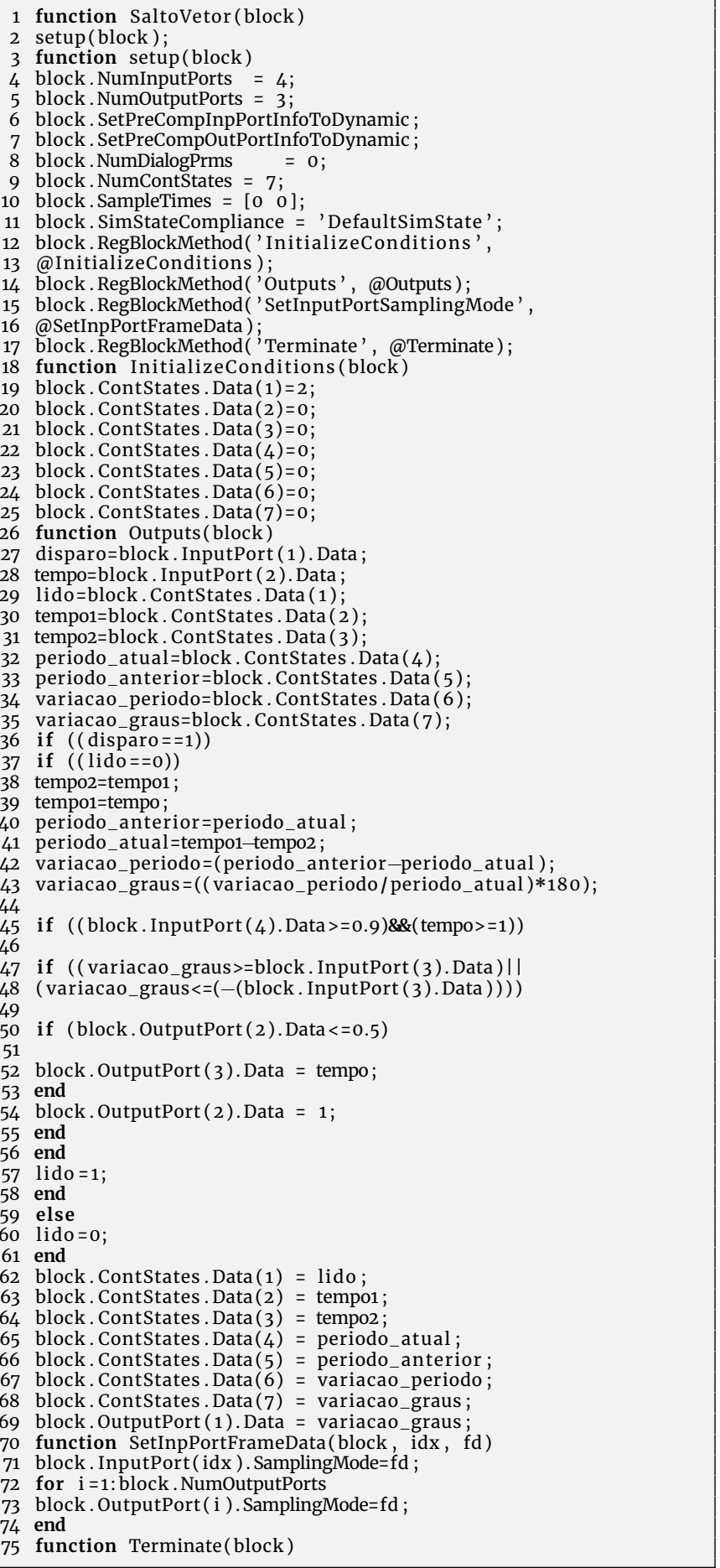

Figura 13: Código $M$ a ser implementado no MATLAB 